\title{
Homophobie und Heterophobie - Schwierigkeiten unterschiedlicher psychosexueller Konstellationen des analytischen Paars ${ }^{1}$
}

\author{
Lothar Schon (München)
}

Zusammenfassung: Der Autor untersucht Auswirkungen von Homophobie und Heterophobie in psychoanalytischen Behandlungen und Supervisionen aus der Perspektive des homosexuellen Analytikers. Anhand einiger Beispiele aus der Zeit während und nach seiner psychoanalytischen Ausbildung demonstriert er die Auswirkungen von Angst vor der psychosexuellen Andersartigkeit des jeweils anderen. Unter anderem betrachtet er die Hartnäckigkeit homophober und (komplementär) heterophober Reaktionen als Folge des jahrzehntelangen Ausschlusses offen homosexueller Menschen von der psychoanalytischen Ausbildung und damit aus der psychoanalytischen Gemeinschaft und ihren Diskursen. Die Öffnung und Liberalisierung der psychoanalytischen Institute gegenüber Homosexuellen eröffnet erst ganz allmählich die Möglichkeit des Austausches über und der Auseinandersetzung mit tiefsitzenden, oft unbewussten Ängsten und Vorurteilen, die aber erstaunlich wenig genutzt wird. Die vorliegende Arbeit möchte einen Beitrag leisten zu einer offenen und allmählich angstfreieren Auseinandersetzung mit Homophobie und Heterophobie in der Psychoanalyse.

Schlüsselwörter: Homophobie, Heterophobie, psychoanalytische Ausbildung, Bisexualität

\section{$1 \quad$ Einleitung}

Als ich mich 2011 an meinem psychoanalytischen Institut darum bewarb, Lehranalytiker zu werden, stellte ich als offen homosexuell lebender Analytiker meine langjährige psychoanalytische Arbeit mit einer heterosexuellen Patientin vor. Die Diskussion verlief lebhaft, kontrovers und in sehr wohlwollender Atmosphäre. Sie war für mich inhaltlich bereichernd, sparte allerdings heikle Fragen der psychosexuellen Paarung in dieser Analyse vollständig aus. Am Schluss hatte ich den Eindruck, meine Bewerbung zum Lehranalytiker sei erfolgreich verlaufen. ${ }^{2}$ Nach dem Ende der Diskussion nahm mich eine erfahrene Lehranalytikerin beiseite und 
konfrontierte mich überraschend damit, dass sie eine ganz andere Sicht auf den Fall habe als die grosse Diskussionsrunde. Sinngemäss sprach sie von der Gefahr, dass homosexuelle Analytiker heterosexuelle Patientinnen nicht wirklich gut psychoanalytisch behandeln könnten, da sie deren (heterosexuelles) Begehren nicht beantworten würden. Sie vertrat konkret die Auffassung, meine Patientin könne durch unsere spezielle psychosexuelle Konstellation retraumatisiert werden. - Der Vater der Patientin war plötzlich verstorben, als das kleine Mädchen zwei Jahre alt war. Die Patientin hatte sich zu Beginn der Behandlung kurz in mich verliebt und dies kommentiert mit den Worten «Aber für mich sind Sie sowieso schwul!», bevor ihre Verliebtheit zunächst sang- und klanglos wieder verschwand. - Die Kollegin problematisierte das seinerzeit aktuelle Erleben der Patientin, dass ich als Mann (bzw. in der Übertragung auch als Vater) aufgrund meiner Homosexualität ebenso «unerreichbar» für sie sei wie der Vater nach seinem plötzlichen Tod.

Ich war doppelt irritiert von dieser Rückmeldung: Zum einen befremdete mich die konkretistische Vorstellung meiner Kollegin, unsere Patienten müssten in ihrem psychosexuellen Begehren von uns «beantwortet» (sprich: potenziell wiederbegehrt) werden, weil wir ihnen sonst schaden könnten. Abgesehen davon ist es vielleicht auch eine allzu vereinfachende Vorstellung, zwischen Homo- und Heterosexuellen gäbe es keinerlei Begehren. Zum anderen war ich als selbstreflexiver und mit diesen Fragen inhaltlich seit Jahren intensiv beschäftigter homosexueller Psychoanalytiker betroffen, traurig und auch zornig darüber, dass die Kollegin ihre Sichtweise nicht in die Diskussion im Plenum eingebracht hatte. Denn natürlich mache ich mir in jeder Behandlung Gedanken darüber, was meine vom Patienten phantasierte und/oder realistisch wahrgenommene psychosexuelle Identität für ihn oder sie bedeuten könnte, und diese Reflexionen bestimmen sehr stark meine Arbeit mit Patienten unterschiedlichen Geschlechts und unterschiedlicher Geschlechtspartnerorientierung. ${ }^{3}$ Insofern hätte ich eine offene Diskussion der von der Kollegin aufgeworfenen Fragen im Plenum sehr begrüsst und für alle lohnenswert gefunden. Die Kollegin mag aber das Gefühl gehabt haben, es sei besser, mir ihre Überlegungen «privat» mitzuteilen, da sie wohl fürchtete, mir mit deren öffentlicher Äusserung zu schaden. ${ }^{4}$

\section{Zur Geschichte der Homosexualität in der Psychoanalyse}

Stakelbeck und Frank (2006) zeichnen in einer komprimierten Übersichtsarbeit das Schicksal psychoanalytischer Theorien zur Homosexualität ebenso nach wie den Umgang mit Homosexuellen in der institutionalisierten Psychoanalyse und in psychoanalytischen Behandlungen. Sie spannen den Bogen von Freuds liberaler, 
wenn auch theoretisch widersprüchlicher Haltung gegenüber Homosexuellen (s.u.) über die jahrzehntelang sich zuspitzende Pathologisierung der Homosexualität insbesondere in der amerikanischen Psychoanalyse bis hin zu der nach erbitterten Diskussionen öffentlich und in den Statuten revidierten Position zunächst wiederum der American Psychoanalytic Association (APA) und schliesslich auch der International Psychoanalytical Association (IPA) in den 90er Jahren des vergangenen bzw. zu Beginn dieses Jahrhunderts.

Freuds ausgesprochen tolerante Haltung gegenüber der Homosexualität zeigt sich u. a. in seiner vielzitierten Äusserung, die heterosexuelle Objektwahl sei ebenso erklärungsbedürftig wie die homosexuelle, womit er beide sexuelle Orientierungen bezüglich Gesundheit oder Pathologie gleichsetzt (Freud, 1905). Vehement antidiskriminierend schreibt er weiter in den Drei Abhandlungen zur Sexualtheorie:

Die psychoanalytische Forschung widersetzt sich mit aller Entschiedenheit dem Versuche, die Homosexuellen als eine besonders geartete Gruppe von den anderen Menschen abzutrennen. (ebd., S. 44)

Solche Zitate zeigen ebenso wie Freuds Brief an die Mutter eines Homosexuellen (Freud, 1960), wie aufgeklärt, modern und emanzipatorisch sich Freud mit dem Phänomen der gleichgeschlechtlichen Liebe auseinandersetzte. Auch institutionell sprach er sich dagegen aus, die Homosexualität von Bewerbern für die psychoanalytische Ausbildung zum Anlass zu nehmen, diese abzulehnen. Stakelbeck und Frank (2006) zitieren einen aus dem Jahr 1921 datierenden Brief Freuds an Ernest Jones, dessen ablehnende Haltung Freud kritisiert:

Your query, dear Ernest, concerning prospective membership of homosexuals has been considered by us and we disagree with you. In effect we cannot agree with their legal prosecution. We feel that a decision in such a case should depend upon a thorough examination of the other qualities of the candidate. (Freud, 1921, zit. n. Lewes, 1988, S. 33)

Wenngleich Freud also die sexuelle Orientierung eines möglichen Ausbildungskandidaten als Entscheidungskriterium verwirft und vielmehr meint, seine bzw. ihre sonstigen persönlichen Qualitäten sollten über eine Aufnahme 
entscheiden, sind seine entwicklungspsychologischen und psychodynamischen Überlegungen zur Entstehung der Homosexualität verwirrend widersprüchlich geblieben:

Unterscheiden lassen sich drei theoretische Positionen: Homosexualität ist in sich selbst pathologisch und impliziert zwangsläufig andere krankhafte Phänomene-Homosexualität ist selbst nicht pathologisch, weist aber als Symptom auf eine Psychopathologie hin - schließlich: Homosexualität ist nicht pathologisch, und ein Zusammenhang mit einer sonstigen Psychopathologie existiert nicht. Zu jeder dieser Positionen finden sich im Freudschen Werk Belege. (Stakelbeck \& Frank, 2006, S. 125)

Freuds Nachfolger, insbesondere die in die USA emigrierten Analytiker der zweiten und dritten Generation, beriefen sich wohl auf die erstgenannte Position. Stakelbeck und Frank zeigen, wie dramatisch die Pathologisierung der Homosexualität sich in den folgenden Jahrzehnten zuspitzte bis hin zur erbitterten Position von Socarides, der bei Homosexuellen generell von einer schwerwiegenden, psychosenahen Entwicklung ausging:

Socarides (...) ist vermutlich der bekannteste Vertreter einer Auffassung, die sich den homosexuellen Mann immer nur am Rande einer emotionalen Katastrophe vorstellen kann. (ebd., S. 126)

Eine solche Haltung zeugt von erschütternder Ignoranz und kann eigentlich nur als Ausdruck massiver Homophobie verstanden werden. Auch Ilka Quindeau (2015) zeichnet in ihrem aktuellen Psyche-Artikel «Recovering from Iatrogenesis... Vom Umgang mit dem homophoben Erbe» die theoretische und klinische Pathologisierung der Homosexualität nach (Quindeau, 2015). Angefangen habe es mit Sandor Radó, der 1940 (kurz nach Freuds Tod also!) die These der konstitutionellen Bisexualität verwarf und Homosexualität konzipierte als «reparativen Ersatz einer angstvoll besetzten Heterosexualität». Ihm folgte 1962 Irving Bieber, der in einer methodisch fragwürdigen klinischen Studie meinte, die typische Familiendynamik des später homosexuellen Mannes gefunden zu haben. Übersehen wurde dabei, dass eine sehr ähnliche Familienkonstellation aus klammernd-intrusiver Mutter, abwesend-feindseligem Vater und einer 
schlechten elterlichen Beziehung auch bei unzähligen heterosexuellen Männern vorkommt. Socarides schliesslich steht für den Höhepunkt der psychoanalytischen Homophobie, da er sich neben wenig überzeugenden psychodynamischen Hypothesen zur Psychogenese der Homosexualität ganz offen hasserfüllt äusserte über Aggression, Destruktion und Selbstbetrug der Homosexuellen, die er als gesellschaftlich geradezu gefährlich betrachtete (vgl. dazu Stakelbeck \& Frank, 2006).

Wenngleich die American Psychoanalytic Association 1991 als erste Fachgesellschaft in einer Antidiskriminierungsklausel Freuds Rat von 1921 (also 70 Jahre später!) endlich umsetzte und die IPA sich dem 2001 um weitere 10 Jahre verspätet anschloss, äussert Quindeau sich skeptisch zum aktuellen Stand der Pathologisierung von Homosexualität in der Psychoanalyse in Deutschland und Europa und meint, die Ausgrenzungspraxis setze sich auf theoretischer, konzeptueller, klinischer und institutioneller Ebene fort (Quindeau, 2015, S. 653). Sie zitiert Shelbys interessante Hypothese, dass die intensive Befassung mit Homosexualität den Blick auf die zugrundeliegende Bisexualität verstellt habe und in diesem Sinne als eine Form von Abwehr verstanden werden könne (a.a. O., S. 657).

Ähnlich wie Quindeau meint Almut Rudolf-Petersen vom Hamburger Institut der Deutschen Psychoanalytischen Gesellschaft, man könne sich bis heute fragen:

War die Streichung der pathologisierenden Passagen aus psychoanalytischen Lehrbüchern Ausdruck einer in der Tiefe veränderten Haltung von PsychoanalytikerInnen oder lediglich ein Zugeständnis an einen gesellschaftlichen Paradigmenwechsel und die ihr folgende Rechtsprechung? War die Pathologisierung nur sang- und klanglos verschwunden, ohne dass falsche Annahmen explizit korrigiert und durch neue Konzepte ergänzt worden waren? (Rudolf-Petersen, 2014)

Die Kollegin bemängelt ebenso wie Ermann (2011) in einem Vortrag über Homosexualität und psychoanalytische Ausbildung, dass im deutschsprachigen Raum bisher erst wenige Arbeiten über die normale homosexuelle Entwicklung und spezielle Übertragungskonstellationen vorliegen. Weiter unten möchte ich eine theoretische Auffassung vorstellen, die Freuds These von der psychischen Bisexualität stringent weiterentwickelt und sie mit der psychoanalytischen Triebtheorie verbindet. Darüber hinaus ermöglicht sie m. E. ein verblüffend einfaches und umfassendes Verständnis von Homophobie, Heterophobie und allen 
anderen phobischen Reaktionen auf die wahrgenommene oder phantasierte psychosexuelle Andersartigkeit des Anderen.

\section{Persönliche Erfahrungen als homosexueller Kandidat während der Ausbildung zum Psychoanalytiker}

Mein allererster Patient während der psychoanalytischen Ausbildung war zufällig ein homosexueller junger Mann. Während der Vorgespräche fragte ich mich, ob es ratsam und sinnvoll sei, meine erste psychoanalytische Behandlung mit einem Mann zu unternehmen, der die gleiche sexuelle Orientierung hat wie ich. Ich empfand wohl Angst, wie das an meinem Institut aufgenommen würde und ob ich mir damit schaden könne. Gleichzeitig nahm ich an, dass ein heterosexueller Kandidat sich eher nicht grundsätzlich fragen würde, ob es ratsam sei, eine heterosexuelle Patientin in Analyse zu nehmen und/oder ob er sich und seiner Ausbildung damit schaden könne. Da ich mich - auch im Hinblick auf dieses Thema - in meiner Lehranalyse sehr gut aufgehoben fühlte, begriff ich die zufällig zustande gekommene analytische Paarung zwischen meinem schwulen Patienten und mir als Chance und Herausforderung, der ich mich stellen wollte. Ich machte dann in dieser Behandlung zwei sehr erstaunliche Erfahrungen: eine mit dem Patienten selbst und eine mit meiner (heterosexuellen) Supervisorin.

Der Patient begegnete mir ausserhalb der Analysestunden relativ häufig. Dies brachte mich innerlich immer wieder sehr in Verlegenheit, denn mein zu Beginn der psychoanalytischen Ausbildung noch starrer und ängstlicher Umgang mit dem Rahmen und den Grenzen der analytischen Beziehung liess mich wünschen, dass solche Begegnungen am besten überhaupt nicht passieren sollten. Manchmal fühlte ich mich von meinem Patienten geradezu verfolgt, obwohl ich mit einiger Sicherheit wusste, dass er mich nicht verfolgte. Solange wir uns in der Tram, in der U-Bahn oder auf der Strasse trafen, liess sich mit diesen Begegnungen äusserlich gut umgehen. Wir waren beide verlegen, grüssten einander freundlich, und jeder ging seiner Wege. Eines Tages jedoch traf ich meinen Patienten spät abends in einer schwulen Szenekneipe. Ich hatte mir gerade an der Theke ein Bier bestellt, als ich ihn sah. Mir wäre beinahe vor Schreck das Bierglas aus der Hand gefallen! Wiederum grüssten wir uns verlegen, aber freundlich von Ferne, und ich bemühte mich, mein Bier nicht allzu hastig hinunterzustürzen, bevor ich das Lokal wieder verliess. Am nächsten Tag hatten wir eine Analysestunde. Ich war bislang davon ausgegangen, dass mein Patient unbewusst bereits zu Beginn der Behandlung meine sexuelle Orientierung wahrgenommen haben müsse und bereitete mich darauf vor, dass er nach der Begegnung im Szenelokal meine für ihn nun offen 
sichtbar gewordene schwule Identität ansprechen würde. Bis dahin hatte er mich in seinem bewussten Erleben durchgehend als heterosexuell phantasiert, was mich als Übertragungsphänomen immer wieder beschäftigt hatte. In der Sitzung nach unserer Begegnung im Lokal äusserte sich mein Patient dann sehr bewundernd ob der Tatsache, dass ich als heterosexueller Mann mich in die schwule Szene begeben habe, «um einmal zu sehen, wie das bei den Schwulen so ist» (sprich: um ihn besser verstehen und mich besser in ihn einfühlen zu können). - Hier begegnete mir zum ersten Mal in meiner Position als Analytiker die grosse Macht der Übertragung: Mein Patient, der die eigene Homosexualität zutiefst ablehnte, brauchte mich in seiner Vorstellung als heterosexuellen Mann! Zu Beginn der Behandlung als heterosexuellen Mann, der ihn, den Schwulen, verachten und ablehnen würde. Im Verlauf der Behandlung und ganz explizit nach der Szene-Begegnung dann aber als heterosexuellen Mann, der sich für sein sexuelles Anderssein interessierte, darüber etwas lernen wollte und es wohlwollend annahm, was ihm dabei helfen würde, seine eigene Homophobie zu überwinden. - Im Grunde eine simple Vaterübertragung (zunächst negativ, dann positiv), die mit meiner eigenen psychosexuellen Realität scheinbar wenig zu tun hatte, die der Patient aber entfaltete, ohne sich dabei von mir «stören» zu lassen. - So weit meine erstaunliche Erfahrung mit dem Patienten.

Natürlich berichtete ich meiner Supervisorin recht aufgeregt von der Begegnung mit dem Patienten in der eindeutig schwulen Umgebung und erhoffte mir von ihr Unterstützung im Umgang mit dieser heiklen Szene, die sich in der äusseren Realität abgespielt hatte. Anstatt mit mir die Begegnung und ihre möglichen Implikationen und Auswirkungen auf die Behandlung zu reflektieren, fragte mich die Supervisorin recht unvermittelt, wozu die Schwulenszene eigentlich überhaupt gut sei? Heutzutage könne man sich doch in unserer liberalen Gesellschaft auch anderswo begegnen und finden. - Ich verspürte Befremden und Zurückweisung, versuchte aber zunächst mit einem gewissen Sendungsbewusstsein, meiner Supervisorin zu vermitteln, dass auch homosexuelle Menschen als Angehörige einer gesellschaftlichen Minderheit zuweilen das Gefühl brauchen, «zu Hause» zu sein (unter Ihresgleichen) - ein Gefühl, das Heterosexuelle ja in aller Regel selbstverständlich haben können. Es ging nun aber weiter mit kritischen Statements über die schwule Szene, u. a. mit sehr negativen Äusserungen über den jährlichen Christopher Street Day (die Schwulenparade), für dessen schrille und überzeichnete Elemente meine Supervisorin nur wenig übrig zu haben schien. Allmählich hatte ich den Eindruck einer feindseligen Atmosphäre, was mich erschreckte, aber auch verstörte, hatte ich mich doch bislang bei dieser Supervisorin mit meiner psychosexuellen Identität durchaus angenommen gefühlt. Als sie schliesslich noch 
äusserte, dass sich die heterosexuellen Kandidaten an unserem Institut ja inzwischen geradezu «in der Minderheit fühlen» würden, ${ }^{5}$ begann ich zu ahnen, dass sich in dieser Supervisionssitzung eine tiefsitzende unbewusste Homophobie Bahn gebrochen hatte, die bei aller äusseren und bewussten Liberalität und Akzeptanz an unserem Institut noch längst nicht überwunden sein konnte. Und die ich vermutlich meinerseits zunächst innerlich heterophob beantworten musste. Ich dachte so etwas wie: «Diese Hetero-Frau versteht mich/uns Schwule einfach überhaupt nicht, sie hat keine Ahnung!» - Als die Supervisorin sich nach dieser in meinem Erleben doch recht aggressiven Sitzung gewohnt freundlich von mir verabschiedete, rettete ich meine schwule Selbstachtung mit der kämpferischen Bemerkung: «Ich glaube, wir müssen noch viel miteinander streiten!», woraufhin nun wiederum meine Supervisorin erschrak.

Nachträglich scheint es mir denkbar, dass unbewusst ein Beziehungsdreieck wirksam geworden sein könnte, in dem sich eine heterosexuelle Frau (Supervisorin) in ihrer psychosexuellen Identität von zwei homosexuellen Männern (Patient und Analytiker) abgelehnt und ausgestossen fühlte, worauf sie aggressiv und entwertend reagieren musste. Auf den empfundenen Angriff antwortete mein Unbewusstes wiederum mit einer heterophoben Solidarisierung mit dem Patienten («wir Schwule»), einer inneren Entwertung der heterosexuellen Supervisorin («keine Ahnung») und aggressiven Gefühlen ihr gegenüber («Wir müssen noch viel miteinander streiten!»). Neben den individuellen und teils unbewussten Reaktionen von Ausbildungskandidat und Supervisorin kann man aber die ganze «Szene» (im Sinne des Szenischen Verstehens) auch unter dem Blickwinkel der Frage betrachten, was diese Inszenierung mit dem Patienten, seiner Geschichte und seiner unbewussten Psychodynamik zu tun haben könnte (s.u.). - Leider sind die Supervisorin und ich seinerzeit nie mehr auf das merkwürdige und unangenehme Geschehen in dieser einen Supervisionssitzung zurückgekommen. Vermutlich waren wir alle beide froh und erleichtert, uns wieder in ungefährlichere Gefilde zu begeben, wo wir uns besser vertrugen.

In seiner zu meinem Bedauern nur 160 Sitzungen währenden Analyse setzte der Patient sich mit meiner Hilfe intensiv mit seiner hasserfüllten Beziehung zu einer sehr besitzergreifenden und intrusiven Mutter auseinander und gebrauchte ${ }^{6}$ mich - wie geschildert - in der Übertragung zunächst als ablehnenden heterosexuellen Vater, später dann als wohlwollend annehmenden und akzeptierenden heterosexuellen Vater. Der reale Vater des Patienten war während dessen Jugend verstorben, was der Patient emotional völlig unbewegt erlebt hatte. Gegen Ende der Analyse tauchten dann heftig erotisch aufgeladene Erinnerungen an den Vater 
auf, den der Patient u. a. erregt durchs Schlüsselloch beobachtet hatte, während dieser sich entkleidete. - Es wäre nun also um den homosexuell begehrten Vater und dessen erotisch ersehnte homosexuelle Antwort auf den Sohn gegangen, und entsprechend hätte sich vermutlich auch die Übertragung des Patienten auf mich geändert. Dies muss sehr bedrohlich für ihn gewesen sein. Ganz offen konnte er sagen, dass er sich mit diesem Thema nicht weiter auseinandersetzen wolle. Er sei zufrieden mit dem Ergebnis der Analyse und wolle sie ohne weiteren Verlängerungsantrag beenden. - Natürlich war das eine unvollständige Analyse, und später fragte ich mich immer wieder, ob dies auch einen Zusammenhang mit meiner eigenen Unsicherheit, Befangenheit und Angst als schwul lebender psychoanalytischer Ausbildungskandidat gehabt hatte. Und mit dem möglicherweise gleichfalls angstbesetzten Umgang meiner Ausbilder mit diesem Thema. Dazu ein weiteres Beispiel:

Eine Ausbildungspatientin, etwas älter als ich und seit langem alleinstehend, verliebte sich in der Analyse in mich und sprach das sehr offen an. Ich mochte die Patientin gern, hatte das Gefühl, sie gut zu verstehen und ihr hilfreich sein zu können. Doch angesichts ihrer Verliebtheit empfand ich sofort Erschrecken und Schuldgefühle und fragte mich, ob die Patientin bei einem heterosexuellen Kollegen nicht besser aufgehoben wäre. - Es waren also Schuldgefühle über meine homosexuelle Orientierung, die meinem Erwidern des heterosexuellen Begehrens der Patientin innerlich und symbolisch im Wege standen. Diese Schuldgefühle waren somit Ausdruck meiner eigenen Homophobie und entsprechen exakt jenen Bedenken, die Jahre später meine Lehranalytiker-Kollegin nach meiner Fallvorstellung äussern würde (s. o.). - Natürlich sprach ich meine Schuldgefühle in der Supervision bei einer anderen heterosexuellen Kollegin an und erlebte wiederum eine Enttäuschung und Zurückweisung: Die Supervisorin wischte meine Bedenken beiseite mit der Bemerkung, schliesslich könne kein Patient eine Liebesbeziehung zu seinem Analytiker haben, und es gehe um das Durcharbeiten dieser Frustration. Dabei spiele die reale sexuelle Orientierung des Analytikers keinerlei Rolle. - Die Supervisorin rekurrierte hier auf das allgemeine Abstinenzgebot: «Die Kur muss in der Abstinenz durchgeführt werden» (Freud, 1915a, S. 313) und das symbolisch damit verbundene Inzest-Tabu. - Damit weigerte sie sich aber für mein Empfinden, mit mir gemeinsam die Besonderheiten von Übertragung und Gegenübertragung zwischen einer heterosexuellen Patientin und einem homosexuell lebenden Analytiker zu untersuchen. Ich war enttäuscht und hatte wiederum das Gefühl «Die (diese Hetera!) versteht mich nicht». Nachträglich ist mir klar, dass meine Supervisorin sich bis dahin wohl nicht mit dieser spezifischen 
Konstellation des analytischen Paares auseinandergesetzt hatte, dass sie vermutlich auch bis dahin keinen praktischen Grund gehabt hatte, dies zu tun. Für den Rest meiner Ausbildungszeit vermied ich es, Besonderheiten der psychosexuellen Verstrickungen mit meinen Patienten in der Supervision zu thematisieren - dafür blieben mir nur meine Lehranalyse und die schwul-lesbische Kollegengruppe, der ich inzwischen angehörte (s.o.).

\section{Theoretische Überlegungen und praktische Schlussfolgerungen zu Homo- und Heterophobie und zu unserem psychoanalytischen Umgang damit}

Der Begriff «Homophobie» wurde geprägt von dem US-amerikanischen Psychotherapeuten George Weinberg, der in den 60er Jahren u. a. über seine Beobachtung nachdachte, dass viele heterosexuelle Psychoanalytiker starke negative persönliche Reaktionen zeigten, wenn sie ausserhalb des klinischen Umfeldes mit Homosexuellen zusammentrafen. Viele Jahre später äusserte er in einem Interview mit Gregory M. Herek:

Ich prägte das Wort «homophobia», um auszudrücken, dass es eine Furcht vor Homosexuellen war (....). Es war eine Furcht vor Homosexuellen, welche mit einer Furcht vor Verseuchung verbunden zu sein schien, einer Furcht davor, die Dinge, für die man kämpfte - Heim und Familie - abzuwerten. (Wikipedia, 2015)

Es geht mir in meiner Arbeit bei der Homophobie nicht um eine sozialpsychologische Perspektive auf das weltweit verbreitete gesellschaftliche Phänomen, das vergleichbar ist mit Fremdenfeindlichkeit, Antisemitismus, Hass auf Flüchtlinge usw. Ich betrachte die Homophobie hier also nicht in erster Linie unter soziologischen oder sozialpsychologischen Gesichtspunkten, obwohl ich diese natürlich für enorm wichtig halte. Zur soziopsychoanalytischen Reflexion von Sexualität verweise ich auf den aktuellen Artikel von Böllinger (2015). Die gesellschaftliche Homophobie ist uns allen geläufig, zumal sich in den westlichen demokratischen Gesellschaften diesbezüglich aktuell ein grosserWandel vollzieht. Jüngste Beispiele sind das Urteil des U.S. Supreme Court, der die gleichgeschlechtliche Ehe in den U.S.A. landesweit legalisierte oder der Volksentscheid der Iren, die sich mit überwältigender Mehrheit für die Homo-Ehe aussprachen. Diese Veränderungen hin zu mehr Toleranz sind bewusste gesellschaftliche Vorgänge, vergleichbar der Entscheidung der APA und später auch der IPA, Homosexuelle zur psycho- 
analytischen Ausbildung zuzulassen. Solche gesellschaftlichen, institutionellen und politischen Entscheidungen sind natürlich sehr erfreulich, sie verändern aber nicht automatisch und zwangsläufig individuelle und tief sitzende negative Einstellungen, die entweder ohnehin unbewusst sind oder aber verdrängt werden müssen, wenn sie gesellschaftlich nicht mehr opportun sind.

Ich möchte vielmehr mit Ihnen nachdenken über Homophobie als persönliche unbewusst negative Einstellung a) gegenüber der psychosexuellen Andersartigkeit des Anderen auf der interpersonellen Ebene und b) gegenüber eigenen verworfenen psychosexuellen Strebungen auf der intrapsychischen Ebene. Es geht mir um Phänomene wie die homophoben Äusserungen der in ihrer bewussten Einstellung toleranten Supervisorin im zweiten Fallbeispiel und meine unbewusste heterophobe Reaktion darauf oder die ebenso homophob erscheinenden Gedanken der Lehranalytiker-Kollegin im ersten Fallbeispiel, die mit Sicherheit in ihren bewussten Einstellungen meint, nichts gegen Schwule und Lesben zu haben und diese in psychoanalytischen Behandlungen auch gerne in ihrem Werdegang begleitet. Zu den in diesem Sinne der eigenen bewussten Überzeugung entzogenen und ihr u. U. widersprechenden unbewussten negativen Einstellungen gehört m. E. unbedingt auch die Heterophobie von homosexuellen Männern und Frauen.

Homophob sind wir vermutlich alle, ob wir wollen oder nicht. Wir alle sind (zumindest bislang) heterosexuell gezeugte Wesen und wachsen unter einem heterosexuellen Primat auf, d. h. mit einer heterosexuellen Vorannahme. So wie im Juristischen die Unschuldsvermutung gilt, bis das Gegenteil bewiesen ist, so phantasieren uns unsere Eltern und unsere Umgebung i.d. R. als heterosexuell, bis das Gegenteil nicht mehr zu übersehen ist. Und manchmal selbst dann noch: Als ein schwuler Patient von mir sich mit Anfang 20 endlich outete, sagte seine aufgebrachte Mutter allen Ernstes: «Du bist nicht schwul, ich kenne Dich besser als Du Dich selbst!» Alle Menschen - egal, ob sie später hetero- oder homosexuell werden - entwickeln in Identifikation mit ihren Eltern und deren Erwartungen sowie dem gesellschaftlich heterosexuellen Primat zunächst homophobe Einstellungen. Homosexuelle haben es ab dem Zeitpunkt ihres eigenen Erkennens, schwul oder lesbisch zu sein, darum zunächst sehr schwer, weil sie die eigene Homophobie zumindest bis zu einem gewissen Mass überwinden müssen, um sich nicht ununterbrochen selbst zutiefst abzulehnen. Um diese andere, dem Vorherrschen von Heterosexualität widersprechende Identität zu festigen, müssen schwule Männer und lesbische Frauen nach meiner Überzeugung auch unbewusst heterophobe Einstellungen entwickeln, also negative Gefühle und Gedanken, die sich gegen «die Heteros» richten. Ein kleines Beispiel dafür war meine innere Reaktion auf 
die Äusserungen meiner Supervisorin (s. o.). Eklatantere Beispiele finden sich z. B. im Alltag solcher schwuler Männer, die näheren Kontakt mit Heterosexuellen vermeiden, die sich vollständig in der Gay Community einrichten und die sich nicht einmal mehr von einem heterosexuellen Arzt behandeln oder von einem heterosexuellen Rechtsanwalt juristisch beraten lassen würden. In der psychoanalytischen Praxis könnte sich eine solch ausgeprägte Heterophobie z. B. darin äussern, ganz überwiegend nur noch schwule bzw. lesbische PatientInnen zu behandeln. Mir geht es hier um die subtileren und darum potenziell unbemerkten Äusserungen von Hetero- und Homophobie in psychoanalytischen Behandlungen. Wie können wir diese Phänomene theoretisch verstehen, und wie gehen wir praktisch mit ihnen um?

Zu den vielfältigen revolutionären Ideen Freuds zählt seine Überzeugung, wir alle seien ursprünglich bisexuell sowie polymorph pervers veranlagt. Um es einmal auszubuchstabieren: Jeder von uns trägt in sich von Geburt an die Möglichkeit, männliche Wesen ebenso zu begehren wie weibliche und ausserdem in seiner sexuellen Betätigung aktiv und passiv, sadistisch und masochistisch, exhibitionistisch und voyeuristisch und vieles andere mehr zu sein. Und hier ist noch gar nicht die Rede von Phänomenen wie Transsexualität, gelebter Bisexualität oder einer Objektwahl, die als Pädophilie, Gerontophilie, Nekrophilie usw. in Erscheinung tritt. Ralf Binswanger (2011) beschäftigt sich in einem anspruchsvollen Artikel mit dem Versuch einer Neu-Konzeption des Perversionsbegriffs, auf die hier nur verwiesen, aber nicht näher eingegangen werden kann (s. dazu Binswangers Beitrag in diesem Heft). Äussere Faktoren (z. B. die eigene körperliche Ausstattung oder das gesellschaftliche Klima) ebenso wie innere bringen die meisten von uns dazu, eine stabile und klar begrenzte psychosexuelle Identität zu entwickeln, in der wir genau wissen, was wir sind und was nicht, wen wir begehren und wen nicht, wie wir ihn oder sie begehren und wie nicht und ob und wie wir das alles akzeptabel verwirklichen und leben können. Dabei wird vieles (das Meiste!) verworfen und verdrängt, und um unser fragiles psychosexuelles Selbst zu stabilisieren, müssen wir Gegenbesetzungen vornehmen und künftig das ablehnen, was wir in uns selbst nicht (mehr) zulassen können.

Gsell und Zürcher (2011) haben einen sehr überzeugenden Versuch unternommen, Freuds Theorie der ursprünglichen psychischen Bisexualität begrifflich zu klären und stringent weiterzudenken, sie mit triebtheoretischen Konzepten zu verknüpfen und dabei Widersprüche in der Freudschen Theorie aufzulösen. Dabei greifen sie zum Einen auf die duale Triebtheorie zurück, in der Freud das Vorhandensein libidinöser und aggressiver Triebe postuliert. Zum Anderen ent- 
wirren sie Freuds widersprüchliche Auffassung von Männlichkeit undWeiblichkeit zugunsten der bei Freud bereits angelegten theoretischen Klärung, dass Triebziele entweder aktiver oder passiver Natur seien. Auf der genitalen Stufe der psychosexuellen Organisation bedeutet das aktive Triebziel, das Objekt penetrieren zu wollen, während das passive Triebziel in dem Wunsch besteht, vom Objekt penetriert zu werden. Alle Menschen verfolgen mit Eintritt in die ödipale Entwicklung beide Triebziele und geraten dabei in einen unauflöslichen Konflikt mit ihrer realen körperlichen Ausstattung und deren Möglichkeiten. Mädchen können schlicht und ergreifend physisch nicht penetrieren, während Jungen zumindest genital nicht penetriert werden können. Den phantasmatischen unbewussten Umgang mit den damit verbundenen schweren Frustrationen und Enttäuschungen der jeweiligen Triebziele beleuchtet Judith Le Soldat in ihrer modernen und dennoch stringent triebtheoretischen Entwicklungspsychologie (Le Soldat, 2015).

Auch wenn sich Zürcher und Gsell, die sehr vertraut sind mit Le Soldats Werk, in ihrer Arbeit zur Bisexualität nicht explizit mit Homo- und Heterophobie beschäftigen, liefert ihr differenziertes Modell der Bisexualität m. E. plausible Erklärungsansätze dafür, warum jede(r) von uns im Verlaufe seiner (ihrer) psychosexuellen Entwicklung zum Zwecke der Abwehr eigener (u. a. körperlicher) Unzulänglichkeit und der damit verbundenen Unerfüllbarkeit mancher sexueller Wünsche unbewusst homophobe bzw. heterophobe Einstellungen entwickeln muss. Nach den bisher vorgetragenen Überlegungen möchte ich den Versuch unternehmen, hinsichtlich der Homophobie unterschiedliche unbewusste Motive zu differenzieren:

Das allgemeinste und umfassendste Motiv bieten die Theorien von Le Soldat sowie von Gsell und Zürcher: Es besteht in der unbewussten Abwehr und Ablehnung solcher psychosexueller Triebregungen bzw. Triebziele, die in der eigenen psychosexuellen Entwicklung, ausgehend von der ursprünglichen Bisexualität, verworfen werden mussten. Ein solches Motiv findet man z. B. bei Männern, die durchaus vorbewusste homosexuelle Triebwünsche haben, diese aber strikt ablehnen und für sich verwerfen. Solche Männer sind oft extrem homophob. Ein weiteres Motiv besteht in der bei meiner Supervisorin unterstellten Angst vor dem Ausgeschlossensein, also der Eifersucht: Da haben zwei was miteinander, und ich darf nicht mitmachen - ein klassisch ödipales Motiv. Eine dritte Variante könnte schlichter Neid sein: Homosexuellen, ganz besonders den schwulen Männern, wird unterstellt, ausgesprochen hemmungslos und triebhaft zu sein, es sprichwörtlich immer und überall zu treiben, also sämtliche bürgerlichen Überich-Anforderungen zu missachten und ihre Lüste maximal auszuleben. Wie viele, jahrzehntelang in 
monogamer Beziehung lebende Männer und Frauen mögen sich heimlich hin und wieder danach sehnen, so hemmungslos sein zu können, wie es den Schwulen ganz generell unterstellt wird? Bitte beachten Sie, dass ich hier von wechselseitigen Projektionen spreche und nicht von der Realität. Ein viertes Motiv tauchte bei Socarides auf, es wurde von Weinberg benannt (s. o.) und ist sicher weit verbreitet: die Angst vor «Ansteckung»: Wenn man diesen homosexuellen Unfug zulässt und toleriert, was soll dann aus der geordneten, heterosexuellen und familienfreundlichen Gesellschaft werden? Meine Supervisorin hatte ja die Befürchtung der heterosexuellen Kollegen ausgesprochen, eine Minderheit zu werden: Eine homosexuelle Welle würde sie alle überrollen, die Schwulen übernähmen das Regiment. - In Wirklichkeit kämpfen Schwule und Lesben sehr hartnäckig darum, auch ganz bürgerliche Familien gründen zu dürfen ...

Vermutlich gibt es noch weitere unbewusste Motive für homophobe Einstellungen und Reaktionen - meine vorgeschlagene Liste erhebt keinen Anspruch auf Vollständigkeit. Aber was sind nun die Schlussfolgerungen aus diesen Überlegungen für die psychoanalytische Praxis? Erstens: Wir alle müssen uns eingestehen, unbewusst homophobe bzw. heterophobe (sowie andere phobische) Einstellungen zu haben - also feindselige Gefühle gegenüber jenem psychosexuellen Begehren, das wir selbst für uns einst verworfen haben -, die uns im Umgang mit der Psychosexualität unserer Patienten beeinflussen. Daraus folgt, dass wir diese zweitens in uns aufspüren müssen: An welchen Stellen empfinden wir Widerwillen, Unverständnis, Befremden usw., wenn es um die Sexualität und psychosexuelle Identität unserer PatientInnen geht? Insbesondere, aber nicht nur dann, wenn die Patienten eine andere Geschlechtspartnerorientierung haben als wir. Und drittens: In der Arbeit mit Patienten, deren psychosexuelle Identität und/ oder deren Begehren wir nicht teilen, müssen wir uns daraufhin befragen, ob und warum wir diese Identität/dieses Begehren ablehnen müssen.

Es geht also - wie bei vielen anderen Themen auch - in der Analyse mit unseren Patienten darum, in der Gegenübertragung eigene verleugnete, verworfene, uns fremde oder fremd gewordene Anteile zu untersuchen und nach Möglichkeit durchzuarbeiten. So wie Kernberg sinngemäss äusserte, dass ein Psychoanalytiker nur dann einen Mörder behandeln könne, wenn er Zugang zum Mörder in sich finde, so müssen wir Zugang finden zu unseren verworfenen homo- oder heterosexuellen, aktiven oder passiven Strebungen bzw. unserer Ablehnung derselben, um unsere Patienten nicht unbewusst homo- oder heterophob zu beantworten. 


\section{Literatur}

Binswanger, R. (2011). «Die Neurose ist sozusagen das Negativ der Perversion»Freuds Formel neu interpretiert. Psyche, 65, 673-698.

Böllinger, L. (2015). Soziopsychoanalytische Reflexionen zur gesellschaftlichen Konstruktion von Sexualität und neuen Beziehungsformen. Psyche, 69, 603-631.

Ermann, M. (2011). Männliche Homosexuelle in der psychoanalytischen Ausbildung. Die Institution, der Kandidat und seine Patient(inn)en. Unveröffentlichter Vortrag auf dem Symposion «Homosexualität und psychoanalytische Ausbildung» am Institut für Psychoanalyse und Psychotherapie im Rheinland am 22.1.2011.

Freud, S. (1905). Drei Abhandlungen zur Sexualtheorie. GWV.

Freud, S. (1915a). Bemerkungen über die Übertragungsliebe. GW X, 306-321.

Freud, S. (1960). Brief an eine amerikanische Mutter. In: Sigmund Freud. Briefe 1873-1939. Frankfurt am Main: Fischer.

Gsell, M. \&Zürcher, M. (2011). Licht ins Dunkel der Bisexualität. Psyche, 65, 699-729. Le Soldat, J. (2015). Grund zur Homosexualität. Werkausgabe, Bd. 1. Stuttgart: Fromman-Holzboog.

Lewes, K. (1988). The psychoanalytic theory of male homosexuality. NewYork: Simon and Schuster.

Quindeau, I. (2015). "Recovering from Iatrogenesis...” Vom Umgang mit dem homophoben Erbe. Psyche, 69, 648-660.

Rudolf-Petersen, A. (2014). Homosexualität und psychoanalytische Ausbildung. Homepage des DPG-Instituts Hamburg: http:/ / www.dpg-institut-hamburg. de/index.php/selbstverstaendnis?id=141

Stakelbeck, F. \& Frank, U. (2006). Kommen die neuen psychoanalytischen Theorien zur männlichen Homosexualität nur noch aus Amerika? In Biechele, U., Hammelstein, P. \& Heinrich, T. (Hrsg.), anders verrückt?! Lesben und Schwule in der Psychiatrie. Jahrbuch Lesben - Schwule - Psychologie 2006. Berlin: Pabst.

Wikipedia (2015). Homophobie. https:// de.wikipedia.org/wiki/Homophobie

\section{Anmerkungen}

1 Erstmals öffentlich vorgetragen in der Münchener Arbeitsgruppe der Deutschen Psychoanalytischen Gesellschaft am 11.11.2015.

2 Tatsächlich wurde ich ein halbes Jahr später zum Lehranalytiker und Supervisor ernannt. 
3 Unter anderem war ich mehrere Jahre Mitglied einer Gruppe schwuler und lesbischer KandidatInnen und AnalytikerInnen, in der wir uns sehr intensiv mit diesen Fragen sowohl theoretisch als auch in klinischer Fallarbeit auseinandersetzten. An dieser Stelle möchte ich den Kolleginnen und Kollegen für die mir sehr wichtige und gewinnbringende Zusammenarbeit danken!

4 Ich war der erste Lehranalytiker-Bewerber unseres Instituts, der schon als offen schwul lebender Mann in die Ausbildung aufgenommen worden war. Auch bundesweit bin ich damit vielleicht der erste zum Zeitpunkt seiner Ernennung als homosexuell geoutete Lehranalytiker. Zumindest sind mir bislang keine weiteren Kollegen bekannt.

$5 \quad$ Mein Ausbildungsinstitut, die Akademie für Psychoanalyse und Psychotherapie München, hatte Ende der 80er Jahre deutschlandweit zu den ersten gehört, die offen homosexuell lebende KandidatInnen zur Ausbildung zuliessen, und inzwischen waren wir tatsächlich schon eine ganze Handvoll schwuler Männer, während sich (vorübergehend) weniger heterosexuelle Männer um die Ausbildung bewarben.

$6 \quad$ I. S. v. Winnicotts "use of the object".

\section{Angaben zum Autor}

Lothar Schon, Dr. phil. Dipl. Psych., 1963, ist Diplompsychologe, Psychologischer Psychotherapeut und Psychoanalytiker in eigener Praxis in München. Er ist Mitglied der Deutschen Psychoanalytischen Gesellschaft (DPG) sowie Dozent, Lehranalytiker, Supervisor und stellvertretender Ausbildungsleiter der Akademie für Psychoanalyse und Psychotherapie München (DGPT). An der Ludwig-MaximiliansUniversität München hat er einen Lehrauftrag für Psychoanalyse am Lehrstuhl für Klinische Psychologie und Psychotherapie. 